\title{
FINITE METACYCLIC GROUPS ACTING ON BORDERED SURFACES
}

\author{
by COY L. MAY
}

(Received 6 November, 1992)

1. Introduction. A group is called metacyclic in case both its commutator subgroup and commutator quotient group are cyclic. Thus a metacyclic group is a cyclic extension of a cyclic group, and metacyclic groups are among the best understood of the nonabelian groups. Many interesting groups are metacyclic. For instance, the dihedral groups and the "odd" dicyclic groups are metacyclic; see [4, pp. 9-11] for more examples. Here we shall consider the actions of these groups on bordered Klein surfaces.

The real genus $\rho(G)$ [9] is the minimum algebraic genus of any bordered Klein surface on which $G$ acts. This parameter is called the "real" genus because of the correspondence between compact Klein surfaces and real algebraic curves [1]; the bordered surfaces correspond to curves with real points. The real genus parameter was introduced in [9], and numerous basic results about the parameter were obtained there. In particular, the real genus of each dicyclic group was determined. In addition, McCullough has calculated the real genus of each finite abelian group [12].

There are, of course, other genus parameters for the finite group $G$. The real genus is most closely related to the symmetric genus [15]. The symmetric genus $\sigma(G)$ is the minimum genus of any Riemann surface on which $G$ acts (possibly reversing orientation). Some basic relationships between the symmetric genus and the real genus are in $[9, \S 5]$. The symmetric genus $\sigma(M)$ of each $K$-metacyclic group $M$ was determined in [11].

Here we investigate the problem of calculating the real genus $\rho(M)$ for each finite metacyclic group $M$. We first obtain a good general lower bound for $\rho(M)$. We then determine $\rho(M)$ in the important case where $M$ is $K$-metacyclic. We also calculate $\rho(M)$ in case $M$ is the direct product of a dihedral group and a cyclic group of odd order. In addition, we determine the real genus of the nonabelian groups of order $p q$, where $p$ and $q$ are distinct odd primes. Finally we calculate $\rho(G)$ for each group $G$ with order between 16 and 24.

2. Preliminaries. We shall assume that all surfaces are compact. Let $X$ be a bordered surface; $X$ is characterized topologically by the orientability, the number $k$ of components of the boundary $\partial X$ and the topological genus $p$. The surface $X$ can carry a dianalytic structure [1, p. 46] and be considered a Klein surface or a non-singular algebraic curve over $\mathbf{R}$. Thus the bordered surface $X$ has an algebraic genus $g$, which is given by the following relation:

$$
g=\left\{\begin{aligned}
2 p+k-1 & \text { if } X \text { is orientable } \\
p+k-1 & \text { if } X \text { is non-orientable }
\end{aligned}\right.
$$

The algebraic genus appears naturally in bounds for the order of an automorphism group of a Klein surface ([8] contains the first such example), and the real genus of a group is defined in terms of the algebraic genus.

AMS(MOS) subject classification (1991). Primary 30F50; Secondary 20H10, 57M60.

Glasgow Math. J. 36 (1994) 233-240. 
There are general upper and lower bounds for the real genus of a finite group in terms of its order $[9, \S \S 3,4]$. A consequence of the lower bound is that for each $\rho \geq 2$ there are only finitely many groups of real genus $\rho$. The upper bound will be quite helpful here.

THEOREM A [9]. Let $G$ be a finite group with generators $z_{1}, \ldots, z_{c}$, where o $o\left(z_{i}\right)=m_{i}$. Then

$$
\rho(G) \leq 1+o(G)\left[c-1-\sum_{i=1}^{c} \frac{1}{m_{i}}\right]
$$

Group actions on Klein surfaces have often been studied using non-euclidean crystallographic (NEC) groups; here see [2], an excellent general reference for the work on Klein surfaces. Let $\mathscr{L}$ denote the full group of automorphisms of the open upper half-plane $U$. An NEC group is a discrete subgroup $\Gamma$ of $\mathscr{L}$ (with the quotient space $U / \Gamma$ compact). Associated with the NEC group $\Gamma$ is its signature, which has the form

$$
\left(p ; \pm ;\left[\lambda_{1}, \ldots, \lambda_{t}\right] ;\left\{\left(v_{11}, \ldots, v_{1 s_{1}}\right), \ldots,\left(v_{k 1}, \ldots, v_{k s_{k}}\right)\right\}\right) .
$$

The quotient space $X=U / \Gamma$ is a surface with topological genus $p$ and $k$ holes. The surface is orientable if the plus sign is used and non-orientable otherwise. The ordinary periods $\lambda_{1}, \ldots, \lambda_{t}$ are the ramification indices of the natural quotient mapping from $U$ to $X$ in fibers above interior points of $X$. The link periods $v_{i 1}, \ldots, v_{i s_{i}}$ are the ramification indices in fibers above points on the $i$ th boundary component of $X$. Associated with the signature (2.1) is a presentation for the NEC group $\Gamma$. For more information about signatures, see [7], [14], and [2].

Let $\Gamma$ be an NEC group with signature (2.1) and assume $k \geq 1$ so that the quotient space $U / \Gamma$ is a bordered surface. Then the non-euclidean area $\mu(\Gamma)$ of a fundamental region for $\Gamma$ can be calculated directly from its signature $[14$, p. 235]:

$$
\mu(\Gamma) / 2 \pi=\gamma-1+\sum_{i=1}^{t}\left(1-\frac{1}{\lambda_{i}}\right)+\sum_{i=1}^{k} \sum_{j=1}^{s_{i}} \frac{1}{2}\left(1-\frac{1}{v_{i j}}\right),
$$

where $\gamma$ is the algebraic genus of the quotient space $U / \Gamma$.

An NEC group $K$ is called a surface group if the quotient map from $U$ to $U / K$ is unramified. If the quotient space $U / K$ has a non-empty boundary, then $K$ is called a bordered surface group. Bordered surface groups contain reflections but no other elements of finite order.

Let $X$ be a bordered Klein surface of algebraic genus $g \geq 2$. Then $X$ can be represented as $U / K$ where $K$ is a bordered surface group with $\mu(K)=2 \pi(g-1)$. Let $G$ be a group of dianalytic automorphisms of the Klein surface $X$. Then there exists an NEC group $\Gamma$ such that $K$ is a normal subgroup of $\Gamma$ and $\Gamma / K \cong G$.

If $\Lambda$ is a subgroup of finite index in $\Gamma$, then $[\Gamma: \Lambda]=\mu(\Lambda) / \mu(\Gamma)$. It follows that the genus of the surface $U / K$ on which $G \cong \Gamma / K$ acts is given by

$$
g=1+o(G) \cdot \mu(\Gamma) / 2 \pi
$$

Minimizing $g$ is therefore equivalent to minimizing $\mu(\Gamma)$. The basic approach is to consider the NEC groups $\Gamma$ for which $G$ is a quotient of $\Gamma$ by a bordered surface group and identify the one for which $\mu(\Gamma)$ is as small as possible. 
Here we shall be particularly interested in metacyclic groups. Each has a certain type of presentation. Begin with a cyclic group $H=\left\langle S \mid S^{m}=1\right\rangle$ of order $m$, and suppose $\operatorname{gcd}(m, r)=1$. Then $H$ has an automorphism $S \rightarrow S^{r}$. Adjoin to $H$ an element $T$ of order $n$ that transforms $H$ according to this automorphism. This produces a new group $G$ of order $m n$, with presentation

$$
S^{m}=T^{n}=1, \quad T^{-1} S T=S^{r}
$$

These relations will be consistent in case

$$
r^{n} \equiv 1(\bmod m)
$$

If, in addition,

$$
\operatorname{gcd}(r-1, m)=1,
$$

then both $G^{\prime}$ and $G / G^{\prime}$ are cyclic. We shall denote the group with presentation (2.4) and conditions (2.5) and (2.6) by $\langle m, n, r\rangle$. Each of these groups is metacyclic, and, conversely, every finite metacyclic group is isomorphic to one of these groups [16]. For additional details, see [4, pp. 9-11], [6, pp. 146-148], or [5, pp. 166, 167].

3. A lower bound. Here we establish a useful lower bound for the real genus of a cyclic extension of a cyclic group. The bound will apply, of course, to metacyclic groups.

THEOREM 1. Suppose the group $G$ is an extension of $Z_{n}$ by $Z_{k}, k \geq 3$, and $\rho(G) \geq 2$. Then

$$
\rho(G) \geq \begin{cases}1+n(k-4) / 2 & \text { if } k=2 l, \text { lodd } \\ 1+n(k-2) / 2 & \text { otherwise }\end{cases}
$$

Proof. Let $G$ act on a bordered surface $X$ of algebraic genus $\rho=\rho(G)$. Then represent $X$ as $U / K$ where $K$ is a bordered surface group. Obtain an NEC group $\Gamma$ with signature (2.1) and a homomorphism $\phi: \Gamma \rightarrow G$ onto $G$ such that kernel $\phi=K$. If $z \in \Gamma$, we write $\bar{z}=\phi(z)$. Let $\gamma$ denote the algebraic genus of the quotient space $Y=U / \Gamma$, and simplify the canonical presentation for $\Gamma$ as in $[9, \S 2]$. In this simplified presentation, there must be at least one element with order larger than two, since $\Gamma / K \cong G$ and $G$ is not generated by involutions. It follows (as in [9]) that

$$
\gamma+t \geq 1
$$

(where $t$ is the number of ordinary periods). Now from (2.3)

$$
(\rho-1) / o(G)=\mu(\Gamma) / 2 \pi,
$$

which is given by (2.2). Write $M=\mu(\Gamma) / 2 \pi$; we obtain a lower bound for $M$. If $\gamma \geq 2$, then obviously $M \geq 1$.

First suppose $\gamma=1$. If $t \geq 1$, then easily $M \geq \frac{1}{2}$. Assume $t=0$. Since $M>0$, the quotient mapping $U \rightarrow Y$ must be ramified above $\partial Y$. In this case, there must be at least two link periods equal to $2\left[3\right.$, p. 264], and we have $M \geq 2 \cdot \frac{1}{4}=\frac{1}{2}$.

Now assume $\gamma=0$ so that the quotient space $Y=U / \Gamma$ is the disc $D$. Then $t \geq 1$ with at least one ordinary period larger than two. If $t \geq 3$, then easily $M \geq-1+\frac{2}{3}+2 \cdot \frac{1}{2}=\frac{2}{3}$. 
Suppose next that $t=2$ and the quotient mapping $U \rightarrow D$ is ramified above $\partial D$. Again there are at least two link periods equal to 2 , and we have $M \geq-1+\frac{2}{3}+\frac{1}{2}+2 \cdot \frac{1}{4}=\frac{2}{3}$.

Continue to assume $\gamma=0$ and $t=2$, but now suppose there is no ramification above $\partial D$. Then the group $\Gamma$ has signature $\left(0 ;+;\left[\lambda_{1}, \lambda_{2}\right] ;\{()\}\right)$, where we may take $\lambda_{1} \leq \lambda_{2}$. From (2.2)

$$
M=1-\frac{1}{\lambda_{1}}-\frac{1}{\lambda_{2}} .
$$

The group $\Gamma$ has presentation

$$
x^{\lambda_{1}}=y^{\lambda_{2}}=c^{2}=[c, e]=x y e=1 .
$$

But the only generating reflection $c$ must be in the bordered surface group $K$, and $e$ is redundant. Thus the quotient group $G \cong \Gamma / K$ is generated by $\bar{x}, \bar{y}$. Since $G$ is an extension of $Z_{n}$ by $Z_{k}$, there is also a homomorphism $\alpha: G \rightarrow Z_{k}$ of $G$ onto $Z_{k}$.

First suppose $\lambda_{1}=2$. Assume 4 divides $k$ or $k$ is odd. In either case, $\alpha(\bar{x})$ is a nongenerator of $Z_{k}$ (possibly the identity) so that $o(\alpha(\bar{y}))=k$ and $\lambda_{2}=o(\bar{y}) \geq k$. Now $M \geq(k-2) / 2 k$. Next suppose $k=2 l$, with $l$ odd. Then $o(\alpha(\bar{y})) \geq l$ and $\lambda_{2}=o(\bar{y}) \geq l$. Thus

$$
M \geq \frac{1}{2}-\frac{1}{l} \geq \frac{1}{2}-\frac{2}{k}=(k-4) / 2 k
$$

Next suppose $\lambda_{1}=3$. If also $\lambda_{2}=3$, then $G$ and its quotient $Z_{k}$ are generated by elements of order 3 . Hence $k=3$, and $M=\frac{1}{3}>(k-2) / 2 k=1 / 6$. If $\lambda_{2}=4$, then $k$ must be $3,4,6$, or 12 . Here $k \leq 12$ so that

$$
(k-2) / 2 k=\frac{1}{2}-\frac{1}{k} \leq \frac{1}{2}-\frac{1}{12}=\frac{5}{12}=M .
$$

If $\lambda_{2}=5$, then $k \leq 15$ and in the same way $(k-2) / 2 k \leq M$. If $\lambda_{2} \geq \lambda_{1} \geq 4$, then $M \geq 1-\frac{1}{4}-\frac{1}{4}=\frac{1}{2}$.

Finally suppose $\gamma=0$ and $t=1$. The group $\Gamma$ has signature $(0 ;+;[\lambda] ;\{(C)\})$, and the period cycle $C$ is not empty. Thus

$$
M \geq-1+1-\frac{1}{\lambda}+2 \cdot \frac{1}{4} \geq \frac{1}{2}-\frac{1}{\lambda} .
$$

The group $\Gamma$ is generated by $x, e$ and some reflections, and

$$
x^{\lambda}=x e=1 \text {. }
$$

Thus the quotient group $G$ is generated by one element $(\bar{x})$ plus some involutions; this must also be true for $Z_{k}$. Suppose $k$ is a multiple of 4 . Then in $Z_{k}$, each involution is a nongenerator and so $o(\alpha(\bar{x}))=k$ and $\lambda=o(\bar{x}) \geq k$. Now $M \geq(k-2) / 2 k$. If $k$ is odd, there are no involutions in $Z_{k}$ so that $o(\alpha(\bar{x}))=k$ and again $M \geq(k-2) / 2 k$. Finally suppose $k=2 l$ where $l$ is odd. In this case $o(\alpha(\bar{x})) \geq l$ and $\lambda=o(\bar{x}) \geq l$. Then $M \geq(k-4) / 2 k$.

A review of the calculations together with (2.3) provides the lower bound for $\rho(G)$. 
4. $K$-metacyclic groups. The lower bound of Theorem 1 is attained for the interesting family of $K$-metacyclic groups. A $K$-metacyclic group $M$ is a metacyclic group $\langle p, p-1, r\rangle$, where $p$ is an odd prime and the number $r$ is a primitive root modulo $p$. In other words, the powers of $r$ represent every nonzero residue class of the prime $p$. It is important to note that the isomorphism class of the $K$-metacyclic group $M=\langle p, p-1, r\rangle$ is independent of the parameter $r$. Consequently, the formula for $\rho(M)$ should not involve $r$.

Now let the $K$-metacyclic group $M=\langle p, p-1, r\rangle$ have the presentation (2.4) with conditions (2.5) and (2.6). Every element of the group $M$ can be written in the canonical form $T^{j} S^{i}$ where $0 \leq i<p$ and $0 \leq j<p-1$. The following basic results are quite useful in calculations in $K$-metacyclic groups. They are not hard to establish and also appear in [5, p. 167].

Proposition 1. Let $M$ be the K-metacyclic group $\langle p, q, r\rangle$, where $q=p-1$. If $j \not \equiv 0 \bmod q$, then the order of the element $T^{j} S^{i}$ is $q / \operatorname{gcd}(j, q)$.

Proposition 2. Let $M$ be the $K$-metacyclic group $\langle p, q, r\rangle$, where $q=p-1$, and let $\left\{T^{u_{i}} S^{v_{i}} \mid i=1, \ldots, k\right\}$ be a set of elements of $M$. These elements generate $M$ if and only if $\operatorname{gcd}\left(u_{1}, \ldots, u_{k}, q\right)=1$ and at least two of the elements do not commute.

The groups of low real genus have been classified $[9, \S 6]$. The $K$-metacyclic group $\langle 3,2,2\rangle$ is the dihedral group $D_{3}$ and thus has real genus zero [9, Th. 3]. Here, then, we only need to consider primes $p \geq 5$. None of the groups of real genus one are $K$-metacyclic [9, Th. 4].

THEOREM 2. Let $M$ be the $K$-metacyclic group $\langle p, p-1, r\rangle$, where the prime $p \geq 5$. Then

$$
\rho(M)= \begin{cases}1+p(p-5) / 2 & \text { if } p \equiv 3 \bmod 4 \\ 1+p(p-3) / 2 & \text { if } p \equiv 1 \bmod 4\end{cases}
$$

Proof. Let $M$ have the presentation (2.4) with conditions (2.5) and (2.6). Write $k=p-1$ and let $A=T^{k / 2} S$. Then $A$ and $T$ clearly generate $M$, and $o(A)=2$ by Proposition 1. Using Theorem A we have $\rho(M) \leq 1+p(p-3) / 2$ in any case.

Now suppose that $p \equiv 3 \bmod 4$. Then $p-1=2 l$, where $l$ is odd. Again write $k=p-1$, and let $A=T^{k / 2} S=T^{\prime} S, B=T^{2} S$. By Proposition $1, o(A)=2$ and $o(B)=l$. It is easy to see that $A$ and $B$ do not commute. Then since $(2, l)=1, A$ and $B$ generate $M$ by Proposition 2. Now applying Theorem A yields $\rho(M) \leq 1+p(p-5) / 2$.

In either case the $K$-metacyclic group $M$ is an extension of $Z_{p}$ by $Z_{p-1}$, and Theorem 1 provides the lower bound for $\rho(M)$.

Not surprisingly, there is some similarity between the formula in Theorem 2 and the corresponding formula for the symmetric genus [11]. The symmetric genus $\sigma(M)$ depends on the value of the prime $p$ modulo 8 , however.

5. Other metacyclic groups. Here we consider some additional families of metacyclic groups, direct products of cyclic and dihedral groups and the nonabelian groups of order pq. In each case either the lower bound (3.1) gives the real genus or we can modify the proof of Theorem 1 to improve this bound. 
TheOREM 3. Let $n$ and $m$ be integers such that $n \geq 3, m \geq 3$, and $m$ is odd. Then

$$
\rho\left(D_{n} \times Z_{m}\right)= \begin{cases}1+n(m-2) & \text { if } n \text { divides } m \\ 1+n(m-1) & \text { otherwise. }\end{cases}
$$

Proof. Let $G=D_{n} \times Z_{m}$. Then $G$ is an extension of $Z_{n}$ by $Z_{2 m}$, and $\rho(G)$ clearly does not have genus 0 or $1[9, \S 6]$. First assume that $n$ divides $m$. By Theorem 1 , $\rho(G) \geq 1+n(m-2)$. Let $D_{n}$ have presentation

$$
A^{2}=B^{n}=(A B)^{2}=1,
$$

and let $W$ be a generator for $Z_{m}$. Then $(A, 1)$ and $(B, W)$ form a generating set for $G$; the generators have orders 2 and $m$ (since $n$ divides $m$ ). Applying Theorem $\mathrm{A}$ gives $\rho(G) \leq 1+n(m-2)$.

Now assume that $n$ does not divide $m$. We shall use the notation of Theorem 1; we show that two cases in the proof can be eliminated. First suppose $\gamma=0, t=2$, and $\Gamma$ has signature $\left(0 ;+;\left[2, \lambda_{2}\right] ;\{()\}\right)$. Since $Z_{2 m}$ is a quotient of $G, m$ must divide $\lambda_{2}$. Assume $\lambda_{2}=m$. Then the generator $\bar{y}$ has odd order $m$. Let $U$ be the projection of $\bar{y}$ into $D_{n}$. Then $U$ has odd order $l$ in $D_{n}$, where $l$ divides $n$ and $m$, that is, $l$ divides $(n, m)<n$. The subgroup $H=\langle U\rangle$ is normal in $D_{n}$ (from the structure of $D_{n}$ ), and $\bar{y} \in H \times Z_{m}$ of course. But now $o\left(G /\left(H \times Z_{m}\right)\right)=2 n m / l m=2 n / l \geq 4$. This is not possible since $G /\left(H \times Z_{m}\right)$ is generated by the image of the involution $\bar{x}$. Hence $\lambda_{2} \neq m$, so that $\lambda_{2} \geq 2 m$ and $M \geq \frac{1}{2}-1 / 2 m$.

Next suppose $\gamma=0, t=1$ and $\Gamma$ has signature $(0 ;+;[\lambda] ;\{(C)\})$, where the period cycle $C$ has at least two link periods equal to 2 . Again $m$ must divide $\lambda$. Suppose $\lambda=m$ and there were exactly two link periods. Then $\Gamma$ has presentation

$$
x^{m}=x e=\left(c_{i}\right)^{2}=\left(c_{0} c_{1}\right)^{2}=\left(c_{1} c_{2}\right)^{2}=1, \quad e c_{0} e^{-1}=c_{2} .
$$

The bordered surface group $K$ contains reflections, and it follows that some generating reflection $c_{i} \in K$ [7, p. 1198]. Let $V$ be the projection of $\bar{x}$ into $D_{n}$. Then $V$ has odd order in $D_{n}, H=\langle V\rangle$ is a normal subgroup of $D_{n}$, and $\bar{x}, \bar{e} \in H \times Z_{m}$. As before, the quotient group $G /\left(H \times Z_{m}\right)$ has order at least 4. But from (5.2) it is easy to see that $G /\left(H \times Z_{m}\right)$ is generated by the image of an involution. Hence either $\lambda \geq 2 m$ or there are at least three link periods. In either case $M \geq \frac{1}{2}-1 / 2 m$.

Therefore, if $n$ does not divide $m$, we have $M \geq \frac{1}{2}-1 / 2 m$ in all cases in the proof of Theorem 1. In this case, then, by (2.3) $\rho(G) \geq 1+n(m-1)$.

Again let $D_{n}$ have presentation (5.1), and let $W$ be a generator for $Z_{m}$. Then $(A B, 1)$ and $(A, W)$ form a generating set for $G$; the generators have orders 2 and $2 m$. Applying Theorem A gives $\rho(G) \leq 1+n(m-1)$.

The smallest member of this family, $D_{3} \times Z_{3}$, is one of the four groups of real genus 4 $[\mathbf{1 0}]$.

Now let $p$ and $q$ be odd primes such that $q$ divides $p-1$. Then the unique nonabelian group $G_{p q}$ of order $p q$ is the metacyclic group $\langle p, q, r\rangle[13, \mathrm{p} .92]$.

THEOREM 4. Let $G_{p q}$ be the nonabelian group of order $p q$, where $p$ and $q$ are odd primes such that $q$ divides $p-1$. Then

$$
\rho\left(G_{p q}\right)=1+p(q-2) .
$$


Proof. Again we use the notation of Theorem 1. Clearly $\rho\left(G_{p q}\right) \geq 2[9, \$ 6]$. Each element of $G_{p q}$ has order at least $q$, and any generating set for $G_{p q}$ must have at least two elements. In the simplified presentation for the NEC group $\Gamma$, there must be at least two elements with order larger than two. Therefore $\gamma+t \geq 2$. Again let $M=\mu(\Gamma) / 2 \pi$. If $\gamma \geq 2$, then $M \geq 1$. Suppose $\gamma=1$. Then $t \geq 1$, and $M \geq 1-1 / q=(q-1) / q$. Finally let $\gamma=0$. Then $t \geq 2$, and $M \geq-1+2(1-1 / q)=(q-2) / q$. Now from (2.3) we have $\rho\left(G_{p q}\right) \geq 1+p(q-2)$.

Let $G_{p q}=\langle p, q, r\rangle$ have presentation (2.4). Then $T$ and $T S$ are two elements of order $q$ that generate $G_{p q}$, and Theorem A provides the upper bound.

6. Small groups and open problems. The real genus of each group with order less than 16 was determined in [9]. Our results here now complete the calculation up to order 24 , with the exception of order 16. The following table gives $\rho(G)$ and also $\sigma(G)$ for each group $G$ with $16<o(G)<24$ and $\rho(G)>0$. The notation is from [4], and the references concern $\rho(G)$.

GROUPS OF SMALL ORDER WITH POSITIVE REAL GENUS

\begin{tabular}{ccccc}
\hline Order & Group $G$ & $\rho(G)$ & $\sigma(G)$ & References \\
\hline 18 & $Z_{3} \times D_{3}$ & 4 & 1 & Theorem 3, [10, \$5] \\
18 & $((3,3,3 ; 2))$ & 4 & 1 & {$[10, \$ 5]$} \\
18 & $Z_{3} \times Z_{6}$ & 10 & 1 & {$[12$, Th. 2.7] } \\
20 & $Z_{2} \times Z_{10}$ & 1 & 0 & {$[\mathbf{9}$, Th. 4] } \\
20 & $\langle 5,4,2\rangle$ & 6 & 1 & Theorem 2 \\
20 & $\langle 2,2,5\rangle$ & 11 & 1 & {$[9$, Th. 9] } \\
21 & $G_{21}$ & 8 & 1 & Theorem 4 \\
\hline
\end{tabular}

There are many unsolved problems about the real genus parameter. We mention some of the more natural ones related to our work here. Some additional problems are in $[9, \S 8]$.

Problem 1. For each $\rho \geq 5$, classify the groups with real genus $\rho$.

Some comments about the groups of real genus 5 are in $[10, \S 8]$.

Problem 2. Determine $\rho\left(D_{n} \times Z_{m}\right)$ for $m$ even, $m \geq 4$.

There is a problem of this type, of course, for each family of finite groups. Some interesting cyclic extensions of cyclic groups are $Z_{2} \times G_{p q}, Z_{2} \times M$ where $M$ is $K$ metacyclic, and $Z_{2} \times D$ where $D$ is an "odd" dicyclic group.

Problem 3. Find $\rho(G)$ for each group $G$ of order less than 32.

This remains an interesting problem due to the groups of orders 16 and 24 . There are 9 non-abelian groups of order 16 and 12 of order 24. Theorem 3 and the results in [9] and [10] handle some of these groups (and, we might add, all the non-abelian groups of orders 28 and 30). However, quite a bit remains to be done. It might be helpful to first develop some general results about 2-groups acting on bordered surfaces before considering the groups of order 16. 


\section{REFERENCES}

1. N. L. Alling and N. Greenleaf, Foundations of the theory of Klein surfaces, Lecture Notes in Mathematics Vol. 219, (Springer-Verlag, 1971).

2. E. Bujalance, J. J. Etayo, J. M. Gamboa, and G. Gromadzki, Automorphism groups of compact bordered Klein surfaces, Lecture Notes in Mathematics Vol. 1439, (Springer-Verlag, 1990).

3. E. Bujalance and E. Martinez, A remark on NEC groups of surfaces with boundary, Bull. London Math. Soc. 21 (1989), 263-266.

4. H. S. M. Coxeter and W. O. Moser, Generators and Relations for Discrete Groups, Fourth Edition, (Springer-Verlag, 1957).

5. J. L. Gross and S. J. Lomonaco, A determination of the toroidal $K$-metacyclic groups, $J$. Graph Theory 4 (1980), 165-172.

6. M. Hall, The theory of groups (Macmillan, 1959).

7. A. M. Macbeath. The classification of non-Euclidean plane crystallographic groups, Canad. J. Math. 19 (1966), 1192-1205.

8. C. L. May, Automorphisms of compact Klein surfaces with boundary, Pacific J. Math. 59 (1975), 199-210.

9. C. L. May, Finite groups acting on bordered surfaces and the real genus of a group Rocky Mountain J. Math. 23 (1993), 707-724.

10. C. L. May, The groups of real genus four, Michigan Math. J. 39 (1992), 219-228.

11. C. L. May and J. Zimmerman, The symmetric genus of $K$-metacyclic groups (to appear).

12. D. McCullough, Minimal genus of abelian actions on Klein surfaces with boundary, Math Z. 205 (1990), 421-436.

13. J. J. Rotman, The theory of groups (Allyn and Bacon, 1965).

14. D. Singerman, On the structure of non-Euclidean crystallographic groups, Proc. Cambridge Philos. Soc. 76 (1974), 233-240.

15. T. W. Tucker, Finite groups acting on surfaces and the genus of a group, J. Combin. Theory Ser. B 34 (1983), 82-98.

16. H. Zassenhaus, The theory of groups (2nd ed.), (Chelsea, 1958).

Department of Mathematics

TOWSON STATE UNIVERSITY

BALTIMORE,

MARYLAND 21204

U.S.A. 